\title{
Venöse Thromboembolien unter kombinierten oralen Kontrazeptiva - aktueller Stand
}

\author{
Venenthrombosen und Lungenembolien unter kombinierten oralen Kontrazeptiva \\ (KOK) werden seit Mai dieses Jahres erneut breit in den Medien diskutiert. Gemäss \\ neuen Studien liegt das Risiko venöser Thromboembolien (VTE) bei KOK mit Drospi- \\ renon im Direktvergleich zwischen jenem der 2. und der 3. Generations-KOK. Das \\ VTE-Risiko ist während des ersten Anwendungsjahres eines KOK am höchsten. Bei \\ den Spontanmeldungen aus der Schweiz lag in fast $\mathbf{4 0} \%$ der VTE-Fälle mindestens \\ ein Risikofaktor vor. Eine sorgfältige und wiederholte (Familien-) Anamnese ist uner- \\ lässlich.
}

Christian De Geytera, Christoph R. Meier ${ }^{b}$, Danijela Pavelic Ferrettic, Hiu Ying Kwanc, Rudolf Stoller ${ }^{c}$

a Prof. Dr., Abteilungsleiter gyn. Endokrinologie und Reproduktionsmedizin, Universitätsspital Basel, Frauenklinik

b Prof. Dr., Leiter «Basel Pharmacoepidemiology Unit» und Chefapotheker Spital-Pharmazie Universitätsspital Basel

c Swissmedic, Schweizerisches Heilmittelinstitut, Abteilung Arzneimittelsicherheit, Bern

\footnotetext{
* Abkürzungen:

KOK kombiniertes orales Kontrazeptivum

VTE venöse Thromboembolie

TVT tiefe Venenthrombose

KI Konfidenzintervall
}

Korrespondenz:

Dr. Danijela Pavelic Ferretti PhD Medical Reviewer

Swissmedic

Hallerstr. 7

CH-3000 Bern

danijela.pavelic@swissmedic.ch
Im Mai 2009 wurde die Geschichte einer jungen Frau, die unter einem $\mathrm{KOK}^{*}$ mit Drospirenon eine zentrale Lungenembolie mit schwerer hypoxischer Hirnschädigung erlitt, in den Schweizer Medien intensiv diskutiert, wobei das Thema zum Teil unvollständig und verzerrt wiedergegeben wurde. Swissmedic legte am 29. 5. 2009 und am 11.6.2009 auf ihrer Homepage die wichtigsten Fakten zum Risiko von VTE dar. Gemeinsam mit ihrem Human Medicines Expert Committee führte Swissmedic anschliessend eine Analyse der aktuellsten Daten und Studien zum VTE-Risiko durch. Die Ergebnisse, Empfehlungen und die vorgesehenen Massnahmen werden hier vorgestellt.

Die kardiovaskulären Risiken der «Anti-Baby-Pille» wurden bereits kurz nach deren Markteinführung Anfang der Sechzigerjahre bekannt. Durch Reduktion der Östrogendosis konnte in der Folge die Häufigkeit arterieller ischämischer Komplikationen und das Risiko von VTE gesenkt werden. Die KOK der 1. Generation - mit Östrogengehalt $\geq 50 \mu \mathrm{g}$ - werden heute z. B. noch bei gleichzeitiger Therapie mit Enzyminduktoren eingesetzt. Die modernen KOK enthalten weniger als $50 \mu$ g Östrogen (Mikropillen) und werden anhand der Gestagen-Komponente eingeteilt: KOK der 2. Generation enthalten Levonorgestrel, jene der 3. Generation Desogestrel oder Gestoden; zu den «antiandrogenen $\mathrm{KOK}$ » zählen jene mit den neueren Gestagenen Chlormadinon, Drospirenon oder dem lange eingeführten Cyproteronazetat. Die sogenannten Minipillen hingegen sind Monopräparate und enthalten nur eine Gestagenkomponente.

1995/1996 lieferten drei neue epidemiologische Studien [1, 2, 3] übereinstimmend Hinweise, dass nicht nur die Östrogendosis, sondern auch die GestagenKomponente das VTE-Risiko beeinflusst - die KOK der damals neuen 3. Generation gingen mit einem höheren VTE-Risiko einher als jene der älteren 2. Generation. Das seltene, aber potentiell lebensbedrohende
VTE-Risiko wurde damals breit auch in den Medien diskutiert («pill scare»). Die Warnhinweise in Fachund Patienteninformationen wurden stark erweitert. Seit 1998 wird wiederholt über ein höheres VTERisiko unter Einnahme von KOK der 3. Generation und bei Neuanwenderinnen von KOK gesprochen [4, $5,6]$. Die um einen Faktor 1,5 bis 2 erhöhte Thromboembolierate der 3.-Generations-KOK wurde in weiteren Beobachtungsstudien wiederholt bestätigt [7].

\section{Venöse Thromboembolien}

VTE zeigen sich vor allem als tiefe Bein- oder Beckenvenenthrombosen (TVT) mit oder ohne Lungenembolie. Das Risiko einer VTE steigt mit zunehmendem Alter und ist bei Frauen höher als beim Mann. In 1-2\% der Fälle sind VTE tödlich. Die wichtigsten Risikofaktoren in der Gesamtbevölkerung sind: (a) eine kürzlich stattgefundene längere Hospitalisation / Operation, (b) das Vorliegen einer malignen Erkrankung, (c) eine Infektion und (d) ein liegender zentral-venöser Katheter [8]. In Beobachtungsstudien hängt die gemeldete VTE-Inzidenz nicht zuletzt von der Gründlichkeit der Diagnostik ab und ist deswegen in verschiedenen Studien unterschiedlich [9]. Während früher überwiegend die Venographie eingesetzt wurde, wird heute eine TVT mehrheitlich mittels Ultraschall und eine Lungenembolie meist mittels Computertomographie diagnostiziert. Trotz der verfeinerten Diagnostik wurde im Rahmen einer seit über 10 Jahren durchgeführten systematischen Erfassung der VTE-Inzidenz in zwölf Krankenhäusern (Worcester, USA) keine signifikante Zunahme dieser Erkrankung festgestellt [8, 10]. Die altersunabhängige Inzidenz von VTE beträgt somit 11,4 Fälle auf 10000 Personen/Jahr (95\% Vertrauensintervall [KI]: 10,8-12,0).

Bei jungen Frauen ist die Einnahme von KOK ein Risikofaktor für VTE. Die VTE-Inzidenzen sind je nach Studie unterschiedlich, z.B. gemäss einer umfangrei- 
chen Meta-Analyse [9] sowie einer aktuellen Kohortenstudie [11], siehe Tabelle 1. Neben Komplikationen geburtshilflicher Massnahmen sind in entwickelten Ländern VTE die Hauptursache für die maternale Mortalität in Verbindung mit Schwangerschaft und Geburt.

\begin{tabular}{|c|c|c|}
\hline \multicolumn{3}{|c|}{$\begin{array}{l}\text { VTE-Inzidenz bei jungen Frauen gemäss Studien } \\
\text { [9] und [11] }\end{array}$} \\
\hline & $\begin{array}{l}\text { VTE-Inzidenz per } \\
10000 \text { Frauenjahre }(95 \% \mathrm{KI})\end{array}$ & Referenz \\
\hline $\begin{array}{l}\text { Bei Frauen } \\
\text { ohne KOK }\end{array}$ & $1,9(1,2-2,5)$ & [11] \\
\hline $\begin{array}{l}\text { Bei Frauen } \\
\text { mit KOK }\end{array}$ & $\begin{array}{l}2,1(0,8-5,2) \\
7,4(5,2-9,6)\end{array}$ & $\begin{array}{l}{[9]} \\
{[11]}\end{array}$ \\
\hline $\begin{array}{l}\text { Bei Schwan- } \\
\text { geren }\end{array}$ & $10,3(5,5-17,7)$ & [11] \\
\hline
\end{tabular}

Thrombophilien: Bei jungen Frauen wird das Risiko einer VTE unter KOK sehr stark erhöht, wenn eine genetische Veranlagung vorliegt, wie z.B. eine heterozygote Faktor-V-Leiden-Mutation (Prävalenz: $5 \%$ ), ein Antithrombin-Mangel, Protein-S-Mangel (2\%), Prothrombin G20210A (2\%) oder ein Protein-C-Mangel $(0,03 \%)$ [12]. Die Einschätzung des individuellen Risikos einer jungen Frau wird bei der Erstverschreibung eines KOK oder bei Eintritt einer Schwangerschaft derzeit nicht durch eine Laboruntersuchung, sondern durch eine Erhebung der Familienanamnese erfasst. Diese Angaben sind allerdings häufig lückenhaft und sollten wiederholt, bzw. vertieft erhoben werden. Ein systematisches Screening der genetischen Veranlagung einer Thrombophilie wäre heute technisch möglich, wird jedoch aufgrund gesundheitsökonomischer Überlegungen nicht allgemein empfohlen [13]. Darüber hinaus werden auch emotionale Auswirkungen auf die Betroffenen im Falle eines Mutationsnachweises angeführt [14]. Zudem scheint das Wissen um die Veranlagung zur Thrombophilie auch von betreuenden Ärzten häufig nicht genügend berücksichtigt oder gar unkorrekt beurteilt zu werden [15].

\section{Spontanmeldungen aus der Schweiz}

Spontanmeldungen erlauben, frühzeitig Sicherheitssignale, d.h. vermutete neue Risiken, zu erfassen. Da sie aber keine Aussagen über die Häufigkeit unerwünschter Wirkungen und schon gar keine vergleichenden Aussagen dazu gestatten, müssen für einen zuverlässigen Vergleich der Präparategruppen zwingend epidemiologische Studien herangezogen werden (s.u.). Unerwünschte Wirkungen neuer Präparate werden grundsätzlich häufiger berichtet als jene seit Jahren bekannter Medikamente. Mit verbessertem Meldebewusstsein und dem massiven Anstieg der Meldefrequenz in den letzten Jahren wurden Fälle präferentiell zu den neueren Präparaten gemeldet.

Seit Bestehen des behördlichen Pharmacovigilance-Zentrums (1990) bis 2.6.2009 hat Swissmedic
1460 Spontanmeldungen zu hormonalen Kontrazeptiva (inkl. Kombinationspräparate, orale Reingestagenpräparate sowie systemisch wirkende Implantate) erhalten. In dieser Zeit wurden 120 Fälle von VTE gemeldet (Tabelle 2).

\section{Tabelle 2}

Spontanmeldungen (CH) 1990-2.6.2009:

VTE unter hormonalen Kontrazeptiva

\begin{tabular}{llll} 
& Anzahl Fälle & $\begin{array}{l}\text { VTE Latenzzeit } \\
\leq 1 \text { Jahr }\end{array}$ & $\begin{array}{l}\text { Risiko- } \\
\text { faktoren }\end{array}$ \\
\hline $\begin{array}{l}\text { Total VTE } \\
\text { Lungen- } \\
\text { embolien }\end{array}$ & 70 & In $57 \%$ & In $38 \%$ \\
\hline $\begin{array}{l}\text { TVT ohne } \\
\text { Lungen- } \\
\text { embolie }\end{array}$ & 50 & & \\
\end{tabular}

Der grösste Teil der VTE-Fälle (87\%) betraf antiandrogene KOK und solche der 3. Generation. Vergleicht man die «Reporting Ratio» (Anteil der VTE-Meldungen an der Gesamtheit der Meldungen zu einer Präparategruppe), die weniger von Zulassungsjahr und Meldefrequenz abhängt, liegen KOK mit Drospirenon etwas hinter jenen der 3. Generation.

Bei fast $40 \%$ aller VTE-Fälle konnte mindestens ein bekannter Risikofaktor festgestellt werden. 57\% aller gemeldeten VTE ereigneten sich im ersten Anwendungsjahr; mehr als die Hälfte von diesen innerhalb der ersten drei Monate nach Beginn der Einnahme.

Bis am 30.9.2009 erhielt Swissmedic insgesamt zehn Berichte über tödliche Lungenembolien, die mit hormonalen Kontrazeptiva in Verbindung gebracht wurden, d.h. mit antiandrogenen KOK (Cyproteronazetat [n=3], Drospirenon [ $n=2]$, Chlormadinon $[n=1])$, 3. Generations-KOK (Desogestrel $[n=3]$ ) und Reingestagenen (Desogestrel [ $\mathrm{n}=1]$, einer Präparategruppe, die gemäss heutigem Wissensstand das VTE-Risiko nicht erhöht. Die erste ereignete sich 1991, die letzte im September 2009. Die Frauen waren zwischen 18 und 49 Jahre alt, in sechs Fällen unter 30. Bei fünf Betroffenen lag mindestens ein Risikofaktor für VTE vor (Übergewicht, höheres Alter, familiäre Thromboseneigung, lange Flugreise).

\section{Epidemiologische Studien}

Die neusten vier epidemiologischen Studien untersuchen das VTE-Risiko Drospirenon-haltiger KOK (D-KOK), im Vergleich zu anderen hormonalen Kontrazeptiva. [16, 17, 18, 19]. Drei davon sind retrospektive Auswertungen von Krankengeschichten [16, 18, 19], eine ist eine prospektive multizentrische Studie [17], in der die Patientinnen zunächst mittels Fragebogen die auszuwertenden Informationen zu Diagnosen und Expositionen weitgehend selbst lieferten.

Die Autoren der europäischen EURAS-Studie [17] wie auch diejenigen der amerikanischen INGENIXStudie [16] kamen zum Schluss, dass D-KOK gegenüber andern KOK kein erhöhtes VTE-Risiko aufweisen. Bei 
beiden Studien hat man aber nicht nur idiopathische Fälle eingeschlossen, sondern wohl auch solche mit direkten Risikofaktoren, wie z.B. einem vor kurzem erfolgten Trauma oder einem chirurgischen Eingriff. Dies vermindert die Möglichkeit, den Einfluss der KOK auf das VTE-Risiko zu messen, und es reduziert tendenziell eine möglicherweise bestehende Risikoerhöhung durch KOK. In der INGENIX-Studie verglich man zudem das VTE-Risiko unter D-KOK-Einnahme mit demjenigen einer Vergleichsgruppe von Frauen, die «andere KOK» einnahm, ohne diese näher zu definieren. Trotz dieser und anderer relativer Schwächen kann man aus diesen Studien vorsichtig schliessen, dass das VTE-Risiko unter D-KOK wohl im Bereich anderer KOK liegt. Ob das Risiko eher relativ tief und vergleichbar mit demjenigen der KOK der 2. Generation oder allenfalls etwas höher wie bei den KOK der 3. Generation anzusiedeln ist, bleibt aber unbeantwortet.

Zwei neue epidemiologische Studien aus Holland [18] bzw. Dänemark [19] vom August 2009 berichteten hingegen über ein erhöhtes VTE-Risiko in Zusammenhang mit D-KOK, wobei das relative VTE-Risiko zwischen jenem der KOK der 2. und der 3. Generation lag. Die Autoren der Fall-Kontroll-Studie [18] und der Kohortenstudie [19] schlossen, wie bei der EURAS-Studie, auch Nicht-Anwenderinnen von KOK in die Studie ein und verglichen das VTE-Risiko unter verschiedenen KOK mit demjenigen bei Frauen ohne KOK-Einnahme. Die Ergebnisse waren insgesamt konsistent und belegten, dass das VTE-Risiko mit steigendem Alter der Frau und höherem Östrogengehalt der Pille zunimmt, und dass das VTE-Risiko zu Beginn der Einnahme am höchsten ist und danach absinkt. Im Direktvergleich der verschiedenen KOK wurde in beiden Studien gezeigt, dass KOK der 3. Generation gegenüber denjenigen der 2. Generation ein rund 2-fach erhöhtes VTE-Risiko aufweisen. Das VTE-Risiko unter D-KOK lag etwas tiefer als bei den KOK der 3. Generation, aber höher als unter KOK der 2. Generation (Tabelle 3). Limitationen der Studien sind $u$. a. das Nichterfassen von Risikofaktoren wie Übergewicht und (Familien-) Anamnese von VTE $[18,19]$ und die weiten Konfidenzintervalle [18]. Zudem wird diskutiert, ob das VTE-
Risiko der 2.-Generations-KOK in der dänischen Studie $\mathrm{zu}$ tief eingestuft wurde.

Zusammenfassend belegen diese vier epidemiologischen Studien erneut, dass Frauen, die KOK einnehmen, generell ein höheres VTE-Risiko haben als NichtAnwenderinnen, was auch für D-KOK gilt. Was die Einstufung des VTE-Risikos bei D-KOK betrifft, so scheint dieses im Direktvergleich zwischen jenem der KOK der 2. und der 3. Generation zu liegen.

\section{Empfehlungen und Massnahmen}

Familienanamnese/ Bluttests: Bei jungen Anwenderinnen von KOK kommen VTE am häufigsten bei einer genetischen Prädisposition (Thrombophilie) oder bei Frauen mit einem Antiphospholipidsyndrom vor. So ist das VTE-Risiko bei einer Frau mit einer Mutation des Faktor V «Leiden» um das 20Fache erhöht. Das Antiphospholipidsyndrom ist eine Autoimmunerkrankung, welche mit einer erhöhten Thromboseneigung und mit wiederholten Fehlgeburten einhergeht. Am häufigsten tritt es im Rahmen des Krankheitsbildes des Lupus erythematodes auf. Sowohl das Antiphospholipidsyndrom als auch die hereditäre Thrombophilie können anamnestisch erfasst werden und stellen Kontraindikationen für die Verschreibung eines KOK dar. Die aufgrund der gezielten Anamnese gestellte Verdachtsdiagnose beider Veranlagungen kann durch Labortests bestätigt werden.

Verschreiben von KOK: KOK sollen nur nach sorgfältiger Indikationsstellung verschrieben werden. Bei der Verschreibung von D-KOK und 3.GenerationsKOK ist das Risiko von VTE besonders zu beachten. Liegt ein Risikofaktor vor, soll eine nicht-hormonelle Verhütungsmethode in Betracht gezogen werden. D-KOK sollten bevorzugt dann eingesetzt werden, wenn die antiandrogene Partialwirkung von Drospirenon therapeutisch erwünscht ist, d.h. Wunsch zur Kontrazeption bei gleichzeitig vorhandenem Hirsutismus oder Akne. Vom Rauchen ist generell im Hinblick auf die arteriellen Komplikationen unbedingt abzuraten.

Fach- und Patienteninformation: Die Arzneimittelinformation weist ausführlich auf die notwendigen

\begin{tabular}{|c|c|c|c|c|c|}
\hline & $\begin{array}{l}\text { Präparate- } \\
\text { gruppe }\end{array}$ & $\begin{array}{l}\text { EURAS- } \\
\text { Studie* }^{[17]}\end{array}$ & 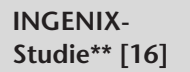 & $\begin{array}{l}\text { Holländische } \\
\text { Studie }{ }^{* * *}[18]\end{array}$ & $\begin{array}{l}\text { Dänische } \\
\text { Studie }^{* *}[19]\end{array}$ \\
\hline Antiandrogene KOK & DRS & 1,0 (Referenz) & $1,0(0,5-1,9)$ & $6,3(2,9-13,7)$ & $1,64(1,27-2,10)$ \\
\hline 2. Generations-KOK & LEV & $1,0(0,6-1,8)$ & -- & $3,6(2,9-4,6)$ & 1,0 (Referenz) \\
\hline \multirow[t]{2}{*}{ 3. Generations-KOK } & GES & -- & -- & $5,6(3,7-8,4)$ & $1,86(1,59-2,18)$ \\
\hline & DES & -- & -- & $7,3(5,3-10,0)$ & $1,82(1,49-2,22)$ \\
\hline «Andere KOK» & & -- & 1,0 (Referenz) & -- & -- \\
\hline Ohne KOK & & -- & -- & 1,0 (Referenz) & -- \\
\hline
\end{tabular}


Vorsichtsmassnahmen hin. Die aktuelle Mediendiskussion zeigt, dass auch die Anwenderinnen sie sorgfältig lesen und Fragen mit ihrer Ärztin/ ihrem Arzt besprechen sollten.

Lungenembolien: Aufgrund der unspezifischen Symptomatik ist es schwierig, eine Lungenembolie frühzeitig zu erkennen. Die häufigsten Anzeichen sind Leistungsabnahme, Dyspnoe und Synkopen. Auch pleuritische Zeichen sollten bei jungen Frauen spezifisch zur Frage nach KOK-Einnahme und Einbezug der Lungenembolie in die Differentialdiagnose führen.

Massnahmen von Swissmedic: Im Rahmen der aktuellen Reevaluation aller KOK hat Swissmedic ein sogenanntes Überprüfungsverfahren für die Drospirenon-haltigen Präparate im Vergleich zu anderen KOK eröffnet. Die wichtigsten Resultate der neuen Studien sollen in deren Arzneimittelinformationen aufgenommen werden.

Meldung vermuteter unerwünschter Wirkungen: Um Probleme wie das dargestellte VTE-Risiko beim alltäglichen Einsatz von Medikamenten weiterverfolgen zu können, ist Swissmedic auf Ihre Meldung angewiesen. Ein gelbes Meldeformular ist diesem Heft beigefügt. Sie finden es auch auf www.swissmedic.ch $(\rightarrow$ Direkt zu $\rightarrow$ Meldungen unerwünschter Wirkungen) oder als letzte Seite des Arzneimittelkompendiums.

\section{Literatur}

1 Jick H, Jick SS, Gurewich V, Myers MW, Vasilakis C. Risk of idiopathic cardiovascular death and non-fatal venous thromboembolism in women using oral contraceptives with differing progestagen components. Lancet. 1995;346:1589-93.

2 Effect of different progestagens in low oestrogen ora contraceptives on venous thromboembolic disease. World Health Organization Collaborative Study of Cardiovascular Disease and Steroid Hormone Contraception. Lancet. 1995;346:1582-8.

3 Spitzer WO, Lewis MA, Heinemann LAJ, Thorogood $\mathrm{M}$, MacRae KD. Third generation oral contraceptives and risk of venous thromboembolic disorders: an international casecontrol study. BMJ. 1996;312:83-8.

4 De Bruijn SF, Stam J, Vandenbroucke JP Increased risk of cerebral venous sinus thrombosis with third-generation oral contraceptives. Cerebral Venous Sinus Thrombosis Study Group. Lancet. 1998;351(9113):1404.

5 Jick SS, Vasilakis C, Jick H. Pregnancies and terminations after 1995 warning about third-generation oral contraceptives. Lancet. 1998;351:1404-5.

6 Herings RMC, Urquhart J, Leufkens HGM. Venous thromboembolism among new users of different oral contraceptives. Lancet. 1999;354:127-8.

7 Maitra NN, Kulier R, Bloemenkamp K, Helmerhors FM, Gülmezoglu AM. Progestogens in combined oral contraceptives for contraception. 2004; Issue 3, CD004861.
8 Spencer FA, Emery C, Joffe SW, Pacifico L, Lessard D, Reed $\mathrm{G}$, et al. Incidence rates, clinical profile, and outcome of patients with venous thromboembolism. The Worcester VTE study. J Thromb Thrombolysis. 2009; Jul 24. [Epub ahead of print].

9 Gomes MPV, Deitcher SR. Risk of venous thromboembolic disease associated with hormonal contraceptives and hormone replacement therapy. Arch Intern Med. 2004;164:1965-76.

10 Spencer FA, Emery C, Lessard D, Anderson F, Emani S Aragam J, et al. The Worcester Venous Thromboembolism study. A population-based study of the clinical epidemiology of venous thromboembolism. J Gen Intern Med. 2006;21:722-7.

11 Samuelsson E, Hägg S. Incidence of venous thromboembolism in young Swedish women and possibly preventable cases among combined oral contraceptive users. Acta Obstet Gynecol Scand. 2004;83:674-81.

12 Andreassi MG, Botto N, Maffei S. Factor V Leiden, prothrombin G20210A substitution and hormone therapy: indications for molecular screening. Clin Chem Lab Med. 2006;44:514-21.

13 Wu O, Robertson L, Twaddle S, Lowe GD, Clark P, Greaves M, et al. Screening for thrombophilia in high-risk situations: systematic review and costeffectiveness analysis. The Thrombosis: Risk and Economic Assessment of Thrombophilia Screening (TREATS) study. Health Technol Assess. 2006;10:1-110.

14 Gartner V, Weber M, Eichinger S. The emotional impact of genetic testing and aspects of counseling prior to prescription of oral contraceptives. Contraception. 2008;78:392-398.

15 Dunn N, White I, Freemantle S, Mann R. The role of prescribing and referral bias in studies of the association between third generation oral contraceptives and increased risk of thromboembolism. Pharmacoepidemiol Drug Saf. 1998;7(1):3-14.

16 Seeger JD, Loughlin J, Eng M, Clifford R, Cutone J, Walker AM. Risk of thromboembolism in women taking ethinylestradiol/drospirenone and other oral contraceptives. Obstet Gynecol. 2007;110:587-93.

17 Dinger JC, Heinemann LA, Kühl-Habich D. The safety of drospirenone-containing oral contraceptive: final results from the European Active Surveillance study on Oral Contraceptives based on 142,475 women-years of observation. Contraception. 2007; 75:344-54.

18 van Hylckama Vlieg A, Helmerhorst FM Vandenbroucke JP, Doggen CJ, Rosendaal FR. The venous thrombotic risk of oral contraceptives, effects of oestrogen dose and progestogen type: results of the MEGA case-control study. BMJ. 2009;339:b2921.

19 Lidegaard Ø, Løkkegaard E, Svendsen AL, Agger C. Hormonal contraception and risk of venous thromboembolism: national follow-up study. BMJ. 2009;339:b2890. 